\title{
Scarring in rheumatoid arthritis and rheumatic heart disease
}

\author{
E. G. L. BYWATERS \\ From the Royal Postgraduate Medical School, London
}

A scar may be defined as imperfect healing or restitution of less than or, sometimes indeed, more than before, usually symbolizing survival. In rheumatic heart disease the initial traumatic lesion of the valve cusp contact areas is first colonized by platelets and fibrin clot; these become gradually more and more fibrotic, partly by surface accretion of fibrin and its replacement by collagen, partly by deeper fibrosis, until we have this big mass of mitral stenosis. A very similar process is seen in lupus erythematosus. How much of this is due to the fibrin deposit associated originally with tissue damage (perhaps due to immune complex activation of complement) and how much due to primary fibroblastic activation associated in rheumatic fever with the original antigenic and presumably streptococcal stimulus we do not know. Current opinion sees Mel Kaplan's cross-reacting streptococcal antibody (Zabriskie et al., 1970) attacking myocardial fibres, but mitral stenosis occurs long after the initial infection and ties up more closely with persistent antibody to group A capsular carbohydrate (Dudding and Ayoub, 1968). Is fibrin laid down faster than it is removed or is there a defect in removal, or is it colonized and collagenized by fibroblasts faster than usual? Or are there defects in its endothelial covering? Is the new collagen type III? Is it more rapidly cross-linked? Do 90000 contractions per day affect stability? Is it undue tension that stimulates the fibroblast or in keloid? There are many more questions. 